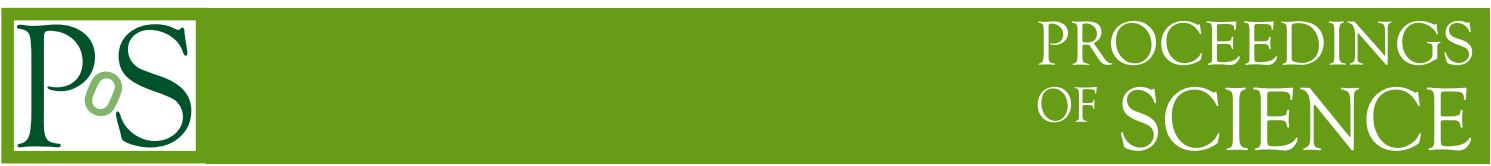

\title{
Gluonic profile of static baryon at finite temperature and the $\mathrm{Y}$ baryonic string
}

\author{
Ahmed S. Bakry*, Derek B. Leinweber, Anthony G. Williams \\ Center for Subatomic Structure of Matter (CSSM), School of Chemistry \& Physics, University of \\ Adelaide, SA, 5005, Australia. \\ E-mail: abakry@physics.adelaide.edu.au
}

\begin{abstract}
The gluon flux distribution of a static three quark system has been revealed at finite temperature in the pure SU(3) Yang-Mills theory. An action density operator is correlated with three Polyakov loops representing the baryonic state at a temperature near the end of the QCD plateau, $T / T_{c} \approx 0.8$, and another just before the deconfinement point, $T / T_{c} \approx 0.9$. The flux distributions display an action-density profile consistent with a filled $\Delta$ shape iso-surface that persists even at large inter-quark separations for both temperatures.

The profile of the baryonic gluonic distribution is compared with the width of the Y-string picture's fluctuations. The comparison reveals that when the distance between at least two quarks is large the best fits are for quantum fluctuations of a Y-shaped baryonic string with the classical junction's position at the Fermat point. This result supports the view that the $\Delta$ shape emerges through the fluctuations of underlying Y-shaped string-like flux tubes.
\end{abstract}

The XXIX International Symposium on Lattice Field Theory - Lattice 2011

July 10-16, 2011

Squaw Valley, Lake Tahoe, California

* Speaker. 


\section{Introduction}

Revealing the color-field distribution in the nucleon is a subject of fundamental importance to our understanding of quantum chromodynamics (QCD) and confinement. Lattice QCD simulations provide a first principles source of knowledge about how the energy distribution manifests itself among a system of three static quarks (3Q). Ambiguities are known to arise, however, in the calculations of the gluonic distribution in the $3 \mathrm{Q}$ system at zero temperature. The energy distribution may be vulnerable to systematic errors associated with excited-state contamination [1] when constructing the static baryon using a Wilson loop operator. The configuration of the spatial links that best minimize the potential has to be adopted before hand. Associated with this arbitrariness in tuning the ground state operator are the excited state potentials which manifest themselves in the revealed gluonic profiles as a bias reflecting the form of spatial links of the Wilson loop operator [1]. The $L$ shape baryon operator provides pronounced evidence where the flux distribution mimics the source [2].

In this investigation, the gluon flux distribution of a three quark system in pure SU(3) YangMills vacuum has been revealed at finite temperature. The static baryonic states are accounted for by means of Polyakov loops. This provides a gauge invariant operator which acquires a methodological importance due to the ability to construct an unbiased 3-quark operator. We have considered two temperatures one at the end of the QCD plateau at $T / T_{c} \approx 0.8$, and one just before the deconfinement point $T / T_{c} \approx 0.9$. This has two advantages: the first is that we can unravel the changes in the shape of the gluonic distribution with the temperature. Indeed, there are significant differences between the behavior of the flux tube just before the deconfinement and at the end of QCD plateau. The second is that the analysis near the end of the QCD plateau may provide insights about the corresponding distribution at zero temperature. This is supported by the small changes in the string tension [3] and admits the possibility of non-uniformity in the ground state gluonic profile [4].

\section{Measurements and statistics}

A scalar field that characterizes the gluonic field can be defined with the action density, $S$, as

$$
\mathscr{C}\left(\vec{\rho}, \vec{r}_{1}, \vec{r}_{2}, \vec{r}_{3}\right)=\frac{\left\langle\mathscr{P}_{3 Q}\left(\vec{r}_{1}, \vec{r}_{2}, \vec{r}_{3}\right) S(\vec{\rho})\right\rangle}{\left\langle\mathscr{P}_{3 Q}\left(\vec{r}_{1}, \vec{r}_{2}, \vec{r}_{3}\right)\right\rangle\langle S(\vec{\rho})\rangle},
$$

with the static baryon constructed by three Polyakov loops

$$
\mathscr{P}_{3 Q}\left(\vec{r}_{1}, \vec{r}_{2}, \vec{r}_{3}\right)=\left\langle P\left(\vec{r}_{1}\right) P\left(\vec{r}_{2}\right) P\left(\vec{r}_{3}\right)\right\rangle
$$

where $\left\langle\ldots . .\right.$. denotes averaging over configurations and lattice symmetries, the vectors $\vec{r}_{i}$ define the positions of the quarks and $\vec{\rho}$ the position of the flux probe. Cluster decomposition implies $C \longrightarrow 1$ away from the quarks. The action density operator is calculated via a highly-improved $\mathscr{O}\left(a^{4}\right)$ three-loop improved lattice field strength tensor [7].

In this investigation, we have taken 10,000 measurements at temperature $T / T_{c} \approx 0.8$, and 6,000 measurements at temperature $T / T_{c} \approx 0.9$. The measurements are taken on hierarchically generated configurations. The gauge configurations are generated using the standard Wilson gauge 
action on lattices with a spatial volume of $36^{3}$. Gauge configurations are generated with a coupling value of $\beta=6.00$. The lattice spacing at this coupling is $a=0.1 \mathrm{fm}$ and the temporal extents are $N_{t}=10$ and $N_{t}=8$ for $T / T_{c} \approx 0.8$ and $T / T_{c} \approx 0.9$, respectively. After each 1000 updating sweeps, $n_{\text {sub }}=20$ or 12 measurements separated by 70 sweeps of updates are taken for the two lattices corresponding to $T / T_{c} \approx 0.8$ and $T / T_{c} \approx 0.9$ respectively. These sub-measurements are binned together in evaluating Eq. (2.1). The total measurements are taken on 500 bins.

\section{Gluon action density}

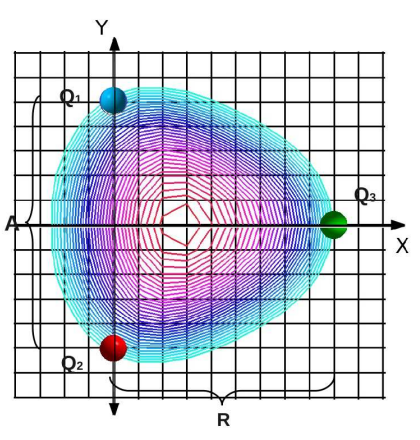

(a) $T \approx 0.8 T_{c}$.

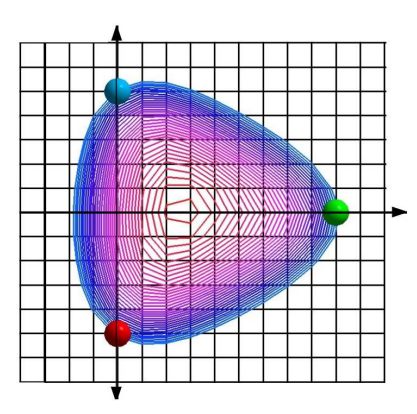

(b) $T \approx 0.9 T_{c}$.

Figure 1: Compares the flux contour lines of the density distribution in the 3Q plane $\mathscr{C}$ for triangular base $A=1.0 \mathrm{fm}$ and third quark separation $R=0.9 \mathrm{fm}$ at $T / T_{c} \approx 0.8$ (Left) and $T / T_{c} \approx 0.9$ (Right).

We have revealed the characteristics of the flux action-density measured for three sets of geometrical 3-quark configurations and the corresponding changes on the behavior due to the temperature. Each set corresponds to isosceles triangle bases of length $A=0.6 \mathrm{fm}, A=0.8 \mathrm{fm}$ and $A=1.0$ $\mathrm{fm}$. The origin of the co-ordinate system is placed at the middle between the two quarks $Q_{2,3}$ on the $y$ axis at positions $\left(0, \pm \frac{A}{2}, 0\right)$ and at distance $R$ from the third quark, $Q_{3}$, at $(x=R, 0,0)$. The quarks reside on the plane $(x, y, 0)$ (Note that $A$ and $R$ are indicated in Fig. 1. The second moment $r_{y}^{2}(x)$ and the amplitude, $H_{y}(x)$, of the flux density at each line $\vec{\rho}\left(x_{i}, y, 0\right)$ is measured by means of Gaussian fits to the complementary distribution $\overline{\mathscr{C}}=1-\mathscr{C}$

$$
\overline{\mathscr{C}}\left(\vec{\rho}\left(x_{i}, y, 0\right)\right)=H_{y}\left(x_{i}\right) e^{-y^{2} / r_{y}^{2}}
$$

The characteristics of the isosurface, the radius and the amplitude profiles of the action density correlations, in addition to the broadening pattern of the flux distribution, can be summarized in the following main points:

A. The iso-surface of the flux action-density displays a family of concave $\Delta$ shapes at small as well as large quark separations. These $\Delta$-shaped gluonic distributions persist and do not change into a Y-shape as the distances between the quark sources are increased. The density plots in the quark plane display a nonuniform distribution at all distance separations as illustrated in Fig. 2. This contrasts the Wilson loop results at zero temperature which exhibit uniform action density along each arm of the Y-shaped profile. A remarkable feature of the revealed map of the contour 


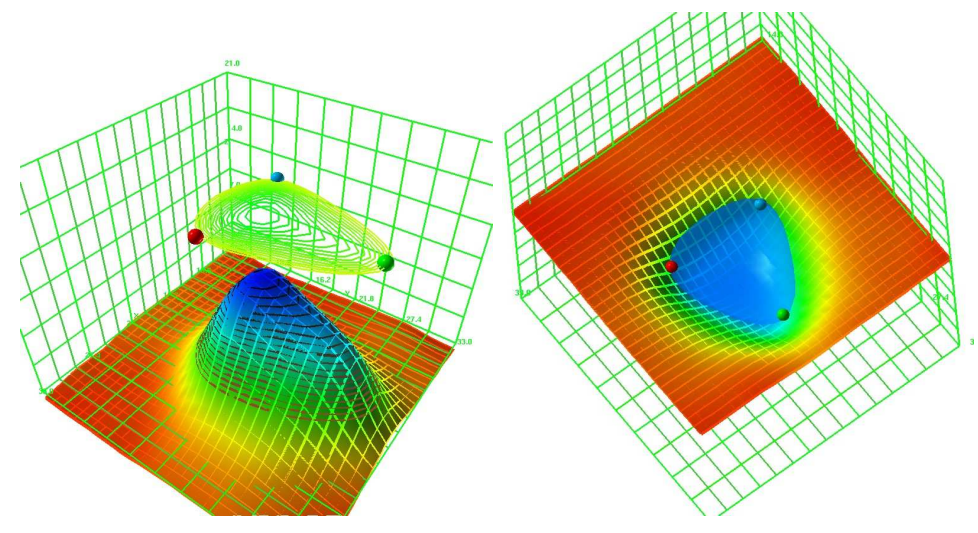

Figure 2: (Left) Surface plot of the action density in the quark plane together with projected contour lines. The $\Delta$-shape is filled with maximum action inside the triangle arrangement near Fermat point. The height and base of the left plot $R=1.2 \mathrm{fm}$ and $A=0.8 \mathrm{fm}$.

(Right) The flux action iso-surface at the quark positions, plotted together with a surface plot for the density distribution $\mathscr{C}(\vec{\rho})$, in the $3 \mathrm{Q}$ plane at temperature $T / T_{c} \approx 0.9$, for equilateral triangular configuration $R=1.1$ fm and $A=1.0 \mathrm{fm}$.

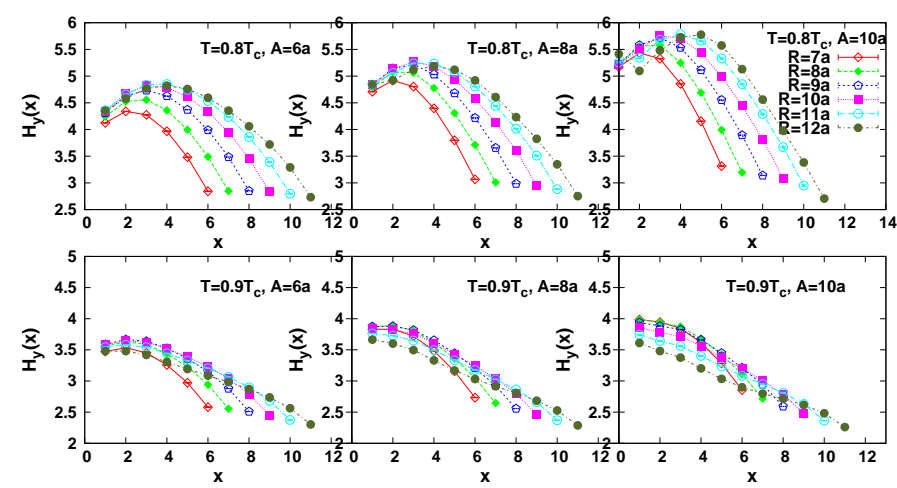

Figure 3: The profile of the action density amplitude, $H_{y}\left(x_{i}\right)$ (scaled by a factor of $10^{1}$ ) for each isosceles configuration with base $A=0.6 \mathrm{fm}, A=0.8 \mathrm{fm}$ and $A=1.0 \mathrm{fm}$, for the two temperatures $T / T_{c} \approx 0.8$ (above), $T / T_{c} \approx 0.9$ (below). The legend signifies the third quark position.

lines of the flux strength is that the shape of the contour lines do not show significant sensitivity to the temperature for the two temperatures considered here.

B. The amplitude profile analysis of the flux density depicted in Fig. 3 shows a maximum vacuum-fluctuation suppression at the plane nearest to the Fermat point of the planar three-quark configurations for intermediate separation distances. The distribution's peak ceases to localize around the Fermat point of the $3 \mathrm{Q}$ isosceles configurations when the height, $R$, is greater than 1.0 $\mathrm{fm}$. The peak shifts to the outside of the triangle made at $T / T_{c} \approx 0.9$ and shifts in the reverse direction to the inside of the triangle for $T / T_{c} \approx 0.8$.

C. The flux mean-square width does not always broaden with the increase of the quark source separation as is the case in the meson. For the lowest temperature, $T / T_{c} \approx 0.8$, the flux 

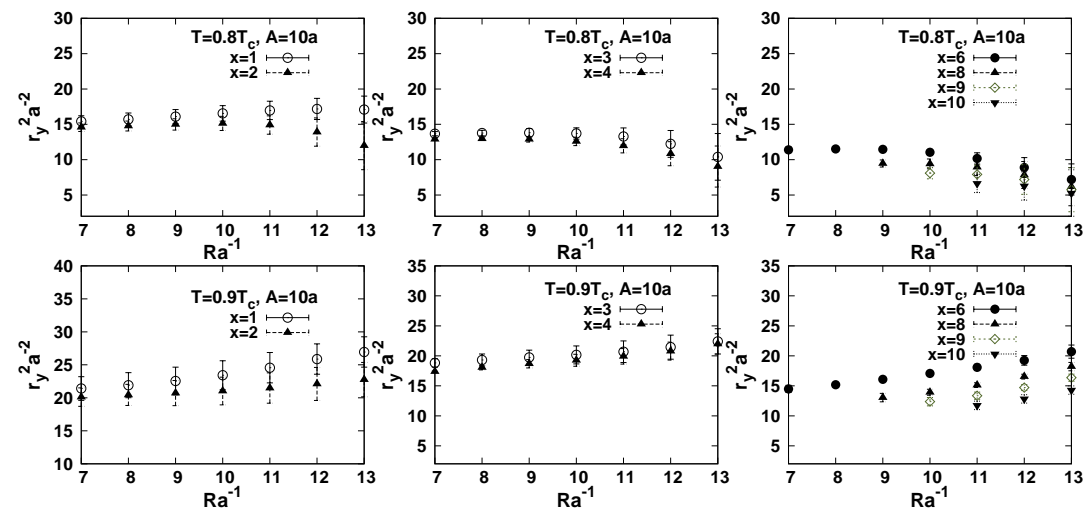

Figure 4: The squared-width of the flux tube at each plane $x_{i}$ versus the third quark separation $R$ for an isosceles base length of $A=1.0 \mathrm{fm}$ at the two temperatures $T / T_{c} \approx 0.8$ (above), $T / T_{c} \approx 0.9$ (below).

distribution shrinks in width for large quark separations. The change in the width of the flux tube shows a non-broadening aspect which is a property of certain configurations of the multi-quark system. The width as depicted in Fig. 4, however, grows linearly near the deconfinement point, $T / T_{c} \approx 0.9$, with the increase of the height of the triangle, $\mathrm{R}$. In general, the slope of the decrease or increase in the width, at both temperatures, depends on the length of the triangle base. The wider the base of the triangle set up by the quark positions, the lower or higher is the slope at temperatures $T / T_{c} \approx 0.8$ and $T / T_{c} \approx 0.9$, respectively.

D. The aspect ratio between the mean square width of the flux distribution in the quark plane and the width in the perpendicular plane exhibits an asymmetry. The gluonic fluctuations in the plane of the quarks are greater than that in the perpendicular directions around Fermat point, indicating a greater restoring force for the system out of the plane of the quarks. This result is consistent with the expectations of the Y-string system.

\section{Baryonic strings}

The analysis of the action density reveals that the observed $\Delta$-shaped flux tube arrangement are not of the shapes of tubes that form a triangle around the perimeter of the three-quark system. In fact the lattice data indicate a filled $\Delta$-shape where the maximal effect on the action density is at the center of the three-quark system (near the Fermat point). Together with the observation of an underlying Y-like shape of the radius profile at large distance, this promotes the understanding that these observed filled $\Delta$ shapes come about through the delocalization of the Y-shaped tube tracing out a filled $\Delta$ shape.

The strong coupling results as well as the lattice results at zero temperature support the Yshaped string picture for the flux tubes in the baryon. In addition to this, the success of the string model near the deconfinement point in the meson indicates that the assumptions of the rough phase of Yang-Mills theory, which justifies the use of the collective co-ordinates for the flux-tubes or the junction, still holds at finite temperatures. This motivates us to consider a generalized Y-shaped baryonic string. In the following, we compare the width of the flux distribution at the Fermat point 
of the triangle set up with the corresponding predictions of the baryonic Y-string model after the inclusion of the thermal effects.
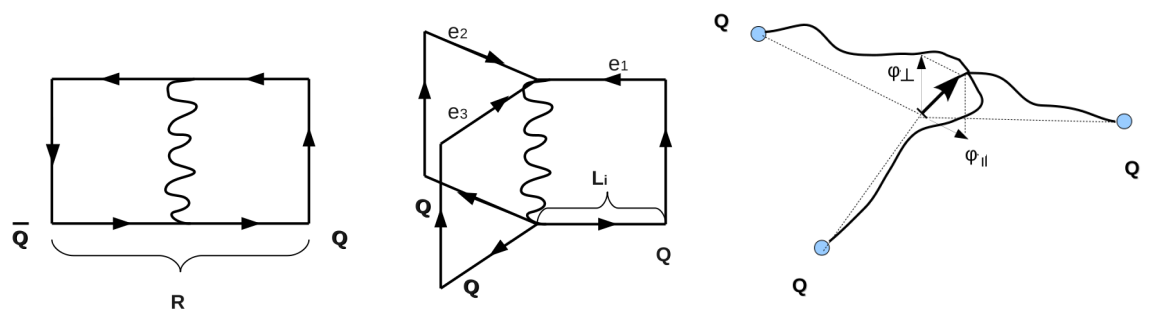

Figure 5: The world sheets of the string in a baryon and a meson. The string in the static meson is modeled as being composed by two strings connected by a junction in the middle.

The thickness of the fluctuating baryonic junction can be calculated based on string model, assuming Nambu Goto action, by evaluating the expectation value

$$
\left\langle\varphi^{2}\right\rangle=\left\langle\varphi_{\perp}^{2}\right\rangle+\left\langle\varphi_{\|}^{2}\right\rangle=\frac{\int \mathcal{D} \varphi \varphi^{2} e^{-S}}{\int \mathcal{D} \varphi e^{-S}} .
$$

The solution of the above integral has been worked out in detail in Ref. [11]. The parallel fluctuations (see Fig. 5) read

$$
\begin{aligned}
\left\langle\varphi_{\|}^{2}\right\rangle & =\frac{1}{\sigma \pi} \sum_{w>0} \frac{1}{w} \frac{k w / \sigma+\left(1-a w^{2}\right) \Theta_{1}}{k^{2} w^{2} / \sigma^{2}+2 k / \sigma w\left(1-a w^{2}\right) \Theta_{1}-4 a w^{2}\left(\Theta_{1}\right)^{2}+\frac{4}{3}\left(1+a w^{2}\right)^{2} \Theta_{2}}+\mu \\
\Theta_{1} & =\frac{1}{3} \sum_{i} \operatorname{coth}\left(w L_{i}\right) \psi\left(w, L_{i}\right) \\
\Theta_{2} & =\frac{1}{3} \sum_{i<j} \sin ^{2}\left(2 \pi \frac{(i-j)}{n}\right) \operatorname{coth}\left(w L_{i}\right) \psi\left(w, L_{i}\right) \operatorname{coth}\left(w L_{j}\right) \psi\left(w, L_{j}\right)
\end{aligned}
$$

with

$$
\psi\left(w, L_{i}\right)=\frac{-k w}{2 \sigma \operatorname{coth}\left(w L_{i}\right)}-\frac{(w T-\pi)}{2 w T \operatorname{coth}\left(w L_{i}\right)}\left(\frac{2 L_{i} \chi\left(\tau_{i}\right)+1}{2 L_{i} \chi\left(\tau_{i}\right)-1}\right)^{w T / \pi-1},
$$

with $\chi(\tau)=\frac{\theta_{2}(0 ; \tau)}{\theta_{1}^{\prime}(0 ; \tau)}, \theta_{i}$ are Jacobi theta functions. The baryonic blades of length $L_{1}, L_{2}, L_{3}$ are measured from the position of the junction $x_{F}$.

We present here our analysis of the width of the action density in the plane of the $3 \mathrm{Q}$ system for $T / T_{c} \approx 0.9$. The measured lattice data are fit to the string model formula of Eq. (4.2). We fit the string model formula Eq. (4.2) to the measured mean square width at the three planes $x=1$, $x=2$ and $x=3$. The returned value of the fit parameters are summarized in Table 1 and Table 2 for isosceles bases $A=0.6 \mathrm{fm}$ and $A=0.8 \mathrm{fm}$, respectively. The closest plane to the Fermat point is $x=2$.

The returned values of the $\chi_{\text {dof }}^{2}$ for the isosceles base of two quarks $A=0.6 \mathrm{fm}$ indicate the acceptable fits to the string model formula are for quark separations $R \geq 0.6 \mathrm{fm}$. The string model 
Table 1: The returned fit parameters of the Y-shaped string model's of Eq. (4.2) for the quark configuration of base $A=6 \mathrm{fm}$, and plane $x=2, T \approx 0.9 T_{c}$.

\begin{tabular}{|c|c|c|c|c|}
\hline \hline Fit range & $\chi^{2}$ & $\chi_{\text {dof }}^{2}$ & $k$ & $\mu$ \\
\hline \hline $4-9$ & 9.11 & 2.27 & 0.01092 & 1.602 \\
$4-11$ & 11.30 & 1.88 & 0.01109 & 1.587 \\
$4-13$ & 11.45 & 1.43 & 0.01110 & 1.588 \\
$6-13$ & 7.02 & 1.17 & 0.01103 & 1.639 \\
\hline \hline
\end{tabular}

Table 2: Same as above table for base $A=8 \mathrm{fm}$, and plane $x=2, T \approx 0.9 T_{c}$.

\begin{tabular}{|c|c|c|c|c|}
\hline \hline Fit range & $\chi^{2}$ & $\chi_{\text {dof }}^{2}$ & $k$ & $\mu$ \\
\hline \hline $4-09$ & 4.04 & 1.01 & 0.00316 & 6.815 \\
$4-13$ & 8.92 & 1.12 & 0.00511 & 6.070 \\
$6-13$ & 3.65 & 0.61 & 0.01168 & 4.259 \\
\hline \hline
\end{tabular}

formula fits the lattice data well for distances $R \geq 0.6 \mathrm{fm}$ reflecting the applicability of the string picture only at large quark separations. Recalling that the Fermat point of this particular configuration lies at $x \approx 1.7$, this is the only plane where Eq. (4.2) can be expected to describe the data well. Indeed, the returned $\chi_{\text {dof }}^{2}$ is much larger than unity at $x=1$ and $x=3$. We conclude that the string picture which indicates the formation of three thin flux tubes that meet at the point that minimize the length of the flux tube is compatible with the lattice data at $T / T_{c} \approx 0.9$. We report the results for $T / T_{c} \approx 0.8$ elsewhere in the near future.

In Table 2, the values also indicate the smallest $\chi_{\text {dof }}^{2}$ occurs at the plane $x=2$ which is the closest plane to the Fermat point of this configuration i.e $x_{F}=2.3$. With the larger base $A=0.8$ fm, smaller values of $R$ provide acceptable fits and we find $R \geq 0.4 \mathrm{fm}$ is acceptable.

\section{References}

[1] F. Okiharu and R. M. Woloshyn, Nuclear Physics B - Proceedings Supplements 129-130, 745 (2004).

[2] F. Bissey et al., Phys. Rev. D 76, 114512 (2007), hep-lat/0606016.

[3] O. Kaczmarek, F. Karsch, E. Laermann, and M. Lutgemeier, Phys. Rev. D 62, 034021 (2000).

[4] A. S. Bakry, D. B. Leinweber, and A. G. Williams, Annals Phys. 326, 2165 (2011), 1102 . 3477.

[5] A. S. Bakry et al., Phys. Rev. D 82, 094503 (2010,hep-lat/1004.0782).

[6] A. S. Bakry, D. B. Leinweber, and A. G. Williams (2011), hep-lat/1107.0150.

[7] S. O. Bilson-Thompson, D. B. Leinweber, and A. G. Williams, Ann. Phys. 304, 1 (2003).

[10] P. J. Moran and D. B. Leinweber, Phys. Rev. D77, 094501 (2008), 0801.1165.

[11] M. Pfeuffer, G. S. Bali, and M. Panero, Phys. Rev. D 79, 025022 (2009). 